\title{
Directed cultivation of Chlorella sorokiniana for the increase in carotenoids' synthesis
}

\author{
Tatiana Kuznetsova ${ }^{1, *}$, Olga Ivanchenko $^{1}$, Elena Trukhina, Maria Nikitina ${ }^{1}$, and Anastasia Kiseleva $^{1}$ \\ ${ }^{1}$ Peter the Great Sankt-Petersburg Polytechnic university, 195251 St. Petersburg, Russian Federation
}

\begin{abstract}
The metabolism of Chlorella sorokiniana is subjected to changes caused by various cultivation conditions. If dosed ultraviolet radiation is used, there is a possibility of a compensatory increase in the synthesis of carotenoids which prevent oxidative stress. Strain 211-8k was cultured under various lighting conditions: control sample was subjected to fluorescent light; sample 1 was subjected to dosed periodic ultraviolet irradiation for 15 minutes every day and fluorescent lighting; sample 2 was subjected to ultraviolet irradiation for 30 minutes in the stabilization phase. Periodic UV exposure negatively affects the population growth of C. sorokiniana which was possible to detect only on the ninth day and the biomass yield significantly decreased. A single UV exposure for 30 minutes lead to a slight decrease in the yield of air-dry biomass which with a further population growth may be compensated. Periodic exposure to UV radiation stimulates carotenoids synthesis, the yield in terms of dry biomass exceeded the control sample on average by $30 \%$. A single ultraviolet irradiation for 30 minutes in the stabilization phase lead to a decrease in the biomass content of both chlorophyll and carotenoids. Microscopy of microalgae showed that ultraviolet radiation leads to the formation of cells with signs of apoptosis.
\end{abstract}

\section{Introduction}

Algae are a unique source of phytochemicals with antioxidant properties [1,2]. Algae differ from terrestrial plants in the biodiversity of carotenoids and their high content [3] as well as there is a possibility of directed carotenogenesis under intensive cultivation of unicellular algae in laboratory conditions. Xanthophylls like astaxanthin, loroxanthin, fucoxanthin, diadinoxanthin, diatoxanthin and siphonin could only be found in algae biomass $[4,5]$.

Microalgae Chlorella sorokiniana is a perspective producer of valuable components that can be successfully cultivated both industrially and in laboratory conditions [6]. C. sorokiniana's biomass is known for a high level of proteins, carbohydrates, lipids and biologically active substances [7].

The content of plastid pigments, chlorophylls and carotenoids (3.5\% in dry biomass) is higher than in terrestrial plants [1]. Chlorophylls and carotenoids are known to have antioxidant properties. Therefore, Chorella's biomass can be used in the production of concentrated pigment complexes and for food production.

According to authors opinion, dosed UV exposure could cause both stimulating and inhibitory effect [8]. Low UV exposure together with photosynthetically active radiation during the cell population growth $\mathrm{C}$. sorokiniana could activate photosensitive mechanisms of autotrophic unicellular algae, which suggests an intensive synthesis of carotenoids [8].
The aim of this study is to describe the effect of dosed UV radiation on the cultivation of $\mathrm{C}$. sorokiniana and to describe the morphological state of the microalgae population and the content of carotenoids in the resulting biomass.

\section{Methods and materials}

\subsection{Cultivation modes of C. sorokiniana}

For the study, the preculture of $C$. sorokiniana (strain 211-8k) from the collection of Algae at Göttingen University (international acronym SAG) was used. The cultivation was carried out in a laboratory bioreactor of a cylindrical shape with a volume of 0.51 [9] using a universal nutrient medium containing all the necessary macro- and micro elements [10].

For the study, the preculture of $C$. sorokiniana (strain 211-8k) from the collection of Algae at Göttingen University (international acronym SAG) was used. The cultivation was carried out in a laboratory bioreactor of a cylindrical shape with a volume of 0.51 [9] using a universal nutrient medium containing all the necessary macro- and micro elements [10].

Mixing was carried out by bubbling with air using a Xilon AP-001 compressor, in the mode of $1.51 / \mathrm{min}$. The cultivation temperature range was $20-23^{\circ} \mathrm{C}$. Mode of illumination was "day-night" (16-8 hours):

- sample 1 is a control sample, daylight lamp (PAR) was used with the intensity of $2500 \pm 200 \mathrm{Lx}, \mathrm{T}(\mathrm{K})$ was 400 ;

\footnotetext{
* Corresponding author: kuznetsova.ta1@ spbstu.ru
} 
- sample 2 is UV1, periodic UV irradiation for 15 minutes per day was carried out with a mercury gas discharge lamp with a spectral region of the light flux of 280-315 nm (UV-B), the intensity was 1300 Lux and further illumination with a fluorescent light (the intensity was $2500 \pm 200$ Lux, T (K) was 400);

- sample 3 is UV2, fluorescent lamp illumination (the intensity was $2500 \pm 200 \mathrm{Lx}$, T (K) was 400$)$, ultraviolet irradiation for 30 min was carried out. (with a spectral region of light flux of 280-315 nm (UV-B), intensity 1300 Lux) at the final phase of cultivation (stabilization).

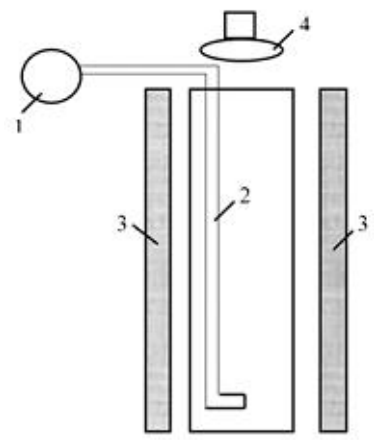

Fig. 1. Laboratory photobioreactor for cultivating the microalga $C$. sorokiniana: 1 - pump-aerator, 2 - air supply tube, 3 - fluorescent lamp, 4 - UV radiation source.

The cultivation was carried out twice. To determine the concentration of cells in suspension during the cultivation of algae, a linear relationship was found between the values of optical density (at $750 \mathrm{~nm}$, in a comparison cuvette nutrient medium) and the concentration of cells in the suspension.

The concentration of cells in the suspension was determined using a hemocytometer, and the optical density was determined on a UNICO 1200/1201 spectrophotometer.

\subsection{Microscopic examination of the C. sorokiniana population}

Microscopy of intravital preparations of a suspension of microalgae was carried out using a MIKMED-6 microscope with a visualization system (JSC LOMO, St. Petersburg).

For processing microphotographs, the FOTO Microanalysis program (LOMO JSC, St. Petersburg) was used. Using Levenguk software (manufactured by Levenhuk LabZZ), the cells (at least 50 pieces) of microalgae were measured in all samples of the study. The long and short axis of the cells were also determined, taking into account the ellipsoidal shape of the cells. The elongation rate $(\mathrm{Ku})$ characterizes the shape of the cells, calculated as the ratio of the long axis of the ellipsoid cell to the short axis.

\subsection{Obtaining the biomass of microalgae $\mathrm{C}$. sorokiniana}

To determine the yield of dry biomass, the separation of the liquid phase from the biomass was carried out by centrifugation in the mode of 5000 vol. / min within 5 minutes with further decantation of the supernatant. The separated biomass was dried in air at $20-23^{\circ} \mathrm{C}$ in a dark place. The moisture content in the air-dry biomass did not exceed $2 \%$

\subsection{Determination of pigments in C. sorokiniana biomass}

Pre-air-dry microalgae biomass was activated mechanically using a high-speed Silent Crusher M homogenizer (IKA® Werke, T25 Basic) at $5000 \mathrm{rpm}$. / min within 2 minutes. $0.025 \mathrm{~g}$ of activated microalgae biomass was poured with $10 \mathrm{ml}$ of $96 \%$ ethanol and ultrasonic extraction of plastid pigments was carried out in the $40 \mathrm{kHz}$ mode for $30 \mathrm{~min}$. The extract was separated from the meal by centrifugation at 3500 vol. / min within 5 minutes The optical density of the extracts was determined spectrophotometrically on a UNICO $1200 / 1201$; pigment absorption bands in the regions of 470,649 , and $664 \mathrm{~nm}$ and the method of the authors [11] [12] were used. The characteristics of the composition of the pigment complex in the obtained extracts were determined by the sum of the pigments and the content of chlorophylls and carotenoids was determined by the sum of the ratio of chlorophylls a and $b$.

The concentrations of chlorophyll a ( $\mathrm{Ch}$ a) and chlorophyll b $(\mathrm{Chb})$ carotenoids $(\mathrm{Cx}+\mathrm{c})$ in the extracts were calculated by using the formulae 1-3 [13]:

$$
\begin{gathered}
\text { Cha }=13.36 A_{664}-5.19 A_{649} \\
\text { Chb }=27.43 A_{649}-8.12 A_{664} \\
C x+c=\left(1000 A_{470}-2.13 \text { Cha }-97.63 \text { Chb }\right) / 209
\end{gathered}
$$

Cha - chlorophyll a concentration $(\mathrm{mkg} / \mathrm{ml})$; $\mathrm{Chb}-$ chlorophyll $\mathrm{b}$ concentration $(\mathrm{mkg} / \mathrm{ml}) ; \mathrm{Cx}+\mathrm{c}-$ carotenoids concentration $(\mathrm{mkg} / \mathrm{ml})$.

A440, A649, A664 - the optical density of the extracts at wavelengths of $470 \mathrm{~nm}, 649 \mathrm{~nm}$ and $664 \mathrm{~nm}$.

The concentration of pigments $\mathrm{A}, \mathrm{mg} / \mathrm{g}$ in terms of the weight of the sample was determined by using the formula (4):

$$
A=\frac{(C \cdot V)}{m \cdot 1000}
$$

$\mathrm{C}$ - pigments concentration, $\mathrm{mg} / \mathrm{1}, \mathrm{V}$ - volume of the extractor hood, $\mathrm{ml}, \mathrm{m}$ - sample mass, $\mathrm{g}$.

\section{Results and Discussion}

\subsection{Cultivation of $\mathrm{C}$. sorokiniana in various light conditions}

At the initial stage of cultivation, the cell concentration in the culture medium was 4.6 million cells / $\mathrm{ml}$ (Fig. 1). The lag phase was not expressed or was no more than 1 day in all samples of the experiment. 


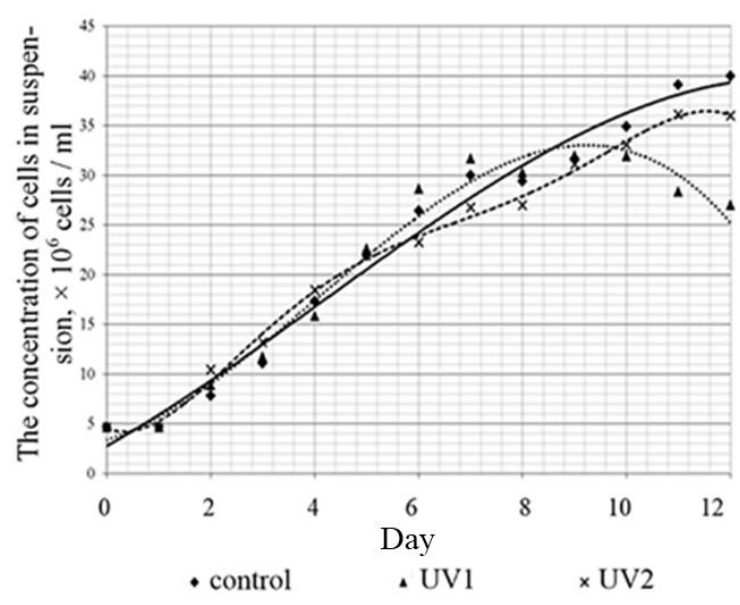

Fig. 2. The yield of air-dry biomass of $C$. sorokiniana obtained by cultivation under various lighting conditions: UV1 periodic ultraviolet irradiation); UV2 - ultraviolet radiation in the stabilization phase.

In the control and UV2 samples, the intensive growth phase was 10-11 days and reduced to 8-9 days in the UV1 sample. A change in the color of the suspension of algae was noted in the stabilization phase in the UV2 sample: it became yellow.

Figure 2 shows the yield of dry biomass obtained during cultivation under different lighting conditions.

With a single UV irradiation (UV2 variant), the biomass yield did not significantly differ from the control sample. With periodic UV irradiation (UV1 variant) the biomass yield has significantly reduced by an average of $48 \%$. The analysis of the cultivation curve showed that a decrease in the biomass content occurred in the final phase of cultivation after 9 days.

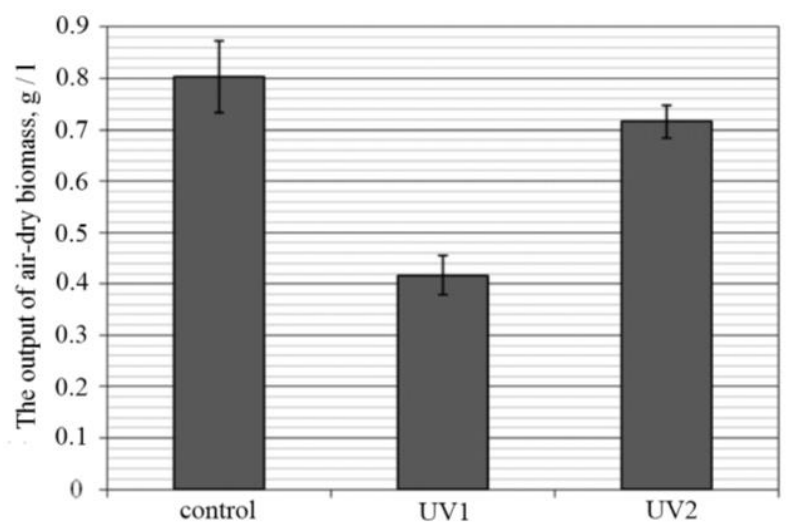

Fig. 3. The yield of air-dry biomass of $C$. sorokiniana obtained by cultivation under various conditions. Illumination: UV1 periodic ultraviolet exposure; UV2 - exposure to ultraviolet light in the stabilization phase.

\subsection{Determination of plastid pigment content in C. sorokiniana biomass}

Figure 4 shows the absorption spectrum of the pigment complex extracted with $96 \%$ ethanol. When analyzing the absorption spectra, two absorption bands were noted in the blue-violet region of $380-500 \mathrm{~nm}$ and in the red region of $640-670 \mathrm{~nm}$. Peaks at 420 (1) and $664 \mathrm{~nm}$ (5) correspond to chlorophyll a [12]. Peaks of 453, $649 \mathrm{~nm}$ correspond to chlorophyll b, shoulder (4) at $420 \mathrm{~nm}$ is described for protochlorophyll [14]. For carotenoids, an absorption band was observed in the region of 420-480 $\mathrm{nm}$ : peaks 440.5 (2) and $470 \mathrm{~nm}$ (3) are described for carotenoids.

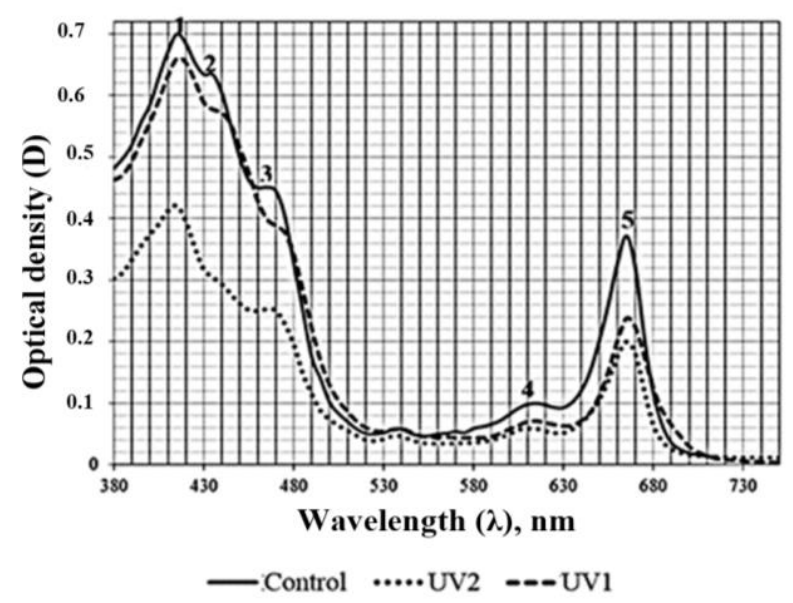

Fig. 4. The absorption spectrum of ethanol extracts of pigments from the air-dry biomass of C. sorokiniana obtained by cultivation under various lighting conditions: control; UV1 periodic ultraviolet exposure; UV2 - exposure to ultraviolet light in the stabilization phase.

Table 1. The content of plastid pigments in the air-dry biomass of $C$. sorokiniana obtained under various cultivation conditions.

\begin{tabular}{|c|c|c|c|}
\hline \multirow{2}{*}{$\begin{array}{c}\text { The pigment } \\
\text { content, mg / g } \\
\text { dry biomass }\end{array}$} & \multicolumn{3}{|c|}{$\begin{array}{c}\text { Biomass samples obtained under } \\
\text { various cultivation conditions }\end{array}$} \\
\cline { 2 - 4 } & control & UV1 & UV2 \\
\hline \multirow{2}{*}{ Cha } & $14.78 \pm$ & $9.94 \pm$ & $13.89 \pm$ \\
& 0.21 & $0.23^{* *}$ & $0.45^{* *}$ \\
\hline \multirow{2}{*}{$C h b$} & $7.73 \pm$ & $4.13 \pm$ & $6.79 \pm$ \\
& 0.17 & $0.11^{* *}$ & $0.35^{* *}$ \\
\hline \multirow{2}{*}{$C x+c$} & $4.24 \pm$ & $5.49 \pm$ & $3.80 \pm$ \\
& 0.14 & $0.10^{* *}$ & $0.12^{* *}$ \\
\hline \multirow{2}{*}{ Pigment amount } & $26.76 \pm$ & $19.56 \pm$ & $24.48 \pm$ \\
& 0.31 & $0.37^{* *}$ & $0.85^{* *}$ \\
\hline \multirow{2}{*}{ Cha / Chb } & $1.92 \pm$ & $2.42 \pm$ & $2.06 \pm$ \\
& 0.05 & $0.05^{* *}$ & 0.05 \\
\hline Note: UV1 - periodic ultraviolet exposure; UV2 - exposure \\
to ultraviolet in the stabilization phase, ** - significant \\
differences at a probability level of 0.99. \\
\hline
\end{tabular}

Long-term UV irradiation caused photodamage of proteins and phospholipids of plasma membranes: oxidation of lipids of membranes by the free radical mechanism with the formation of hydroperoxides with their subsequent photochemical cleavage and obtaining stable final products. It was previously shown that the effect of acute doses of UV radiation on algae cells is accompanied by an increase in the level of protective antioxidant activity of algae [15].

In most cases, photodamage was the result of the generation of an excess of triplet excited Chl capable of interacting with $\mathrm{O} 2$, the product of oxygenated photosynthesis. As a result, chemically reactive forms of singlet $\mathrm{O} 2$ were formed [1]. 
In some cases, carotenoids are an important structural element of transmembrane complexes which along with other components ensure the stability of their structure and the effective fulfillment by the complexes of their main function during photosynthesis [16]. The photoprotective function of carotenoids is associated with their ability to quench the electron excitation energy due to the delocalization of the electron by the conjugated bond system [16].

Periodic UV irradiation leaded to increased carotenogenesis, the amount of carotenoids in the biomass increases by an average of $29.5 \%$ compared to the control sample, with a longer single exposure to UV radiation in the stabilization phase, a decrease in the amount of carotenoids by an average of $10.4 \%$ was observed.

\subsection{Microscopic examination of the C. sorokiniana population in various light conditions}

Figure 5 presents a microscopic picture of the population of $C$. sorokiniana cells in the stabilization phase obtained by periodic (UV1) and single long-term UV irradiation (UV2).

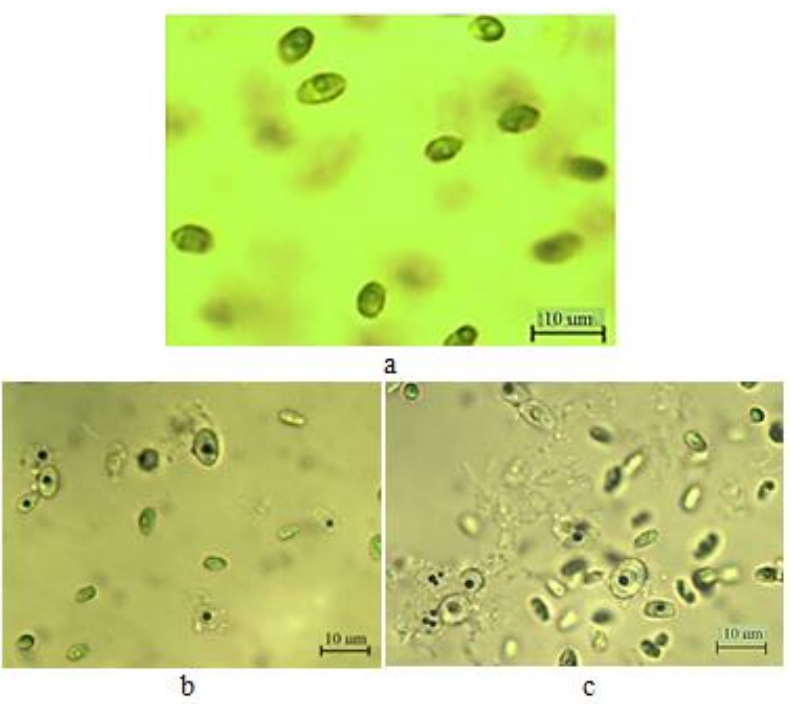

Fig. 5. Microscopic picture of C. sorokiniana populations in the stabilization phase obtained under different cultivation conditions: $\mathrm{a}$ - control; $\mathrm{b}$ - periodic ultraviolet exposure; $\mathrm{c}-$ exposure to ultraviolet light in the stabilization phase.

When studying intravital preparations (in 10 fields of view) in the UV1 (Fig. 5 b) and UV2 (Fig. 5 c) samples, the appearance of bleached cells with a large vacuole and a nucleus with condensed chromatin was noted; morphologically altered cells were larger than the others. The formation of flocks that contribute to the sedimentation of biomass was also noted.

According to published data, the appearance of morphologically altered cells with large vacuoles destroyed by chlorophyll is characteristic of cells under the influence of temperature and osmotic stress factors as well as UV irradiation which is typical for apoptosis [17, $18]$.
A morphometric study of C. sorokiniana microalgae under ultraviolet irradiation showed that the cell size ( $\mathrm{S}$ projection is the projection area of the cells) did not significantly change compared to the control variant (Table 2). However, the elongation coefficient $(\mathrm{Ku})$ of the cells decreased: they became compact and rounded.

Table 2. Morphological characteristics of the cells of the C. sorokiniana population under different cultivation conditions.

\begin{tabular}{|c|c|c|}
\hline Sample & Ku & S projection, $\boldsymbol{\mu m}^{\mathbf{2}}$ \\
\hline control & $1.85 \pm 0.025$ & $23.63 \pm 0.754$ \\
\hline UV1 & $1.66 \pm 0.026^{* *}$ & $22.87 \pm 0.622$ \\
\hline UV2 & $1.72 \pm 0.025^{* *}$ & $24.60 \pm 0.808$ \\
\hline $\begin{array}{l}\text { Note: UV1 - periodic ultraviolet exposure; UV2 - } \\
\text { exposure to ultraviolet in the stabilization phase, } \mathrm{Ku}- \\
\text { cell elongation coefficient; } * *-\text { significant differences } \\
\text { at a probability level of } 0.99 .\end{array}$ \\
\hline
\end{tabular}

\section{Conclusion}

Thus, during periodic UV irradiation, a decrease in the number of algae cells in suspension on day 9 was observed and the biomass yield was significantly reduced compared to the control. A single UV exposure for 30 minutes lead to a slight decrease in the yield of air-dry biomass, which was compensated with a further increase in the population.

Periodic UV irradiation lead to activation of carotenogenesis, the yield of the sum of carotenoids in terms of dry biomass exceeded the control option by an average of $30 \%$.

A single ultraviolet irradiation for 30 minutes during the stabilization phase lead to a decrease in the content of both chlorophyll and carotenoids in the biomass.

Microscopic examination of microalgae populations showed that UV irradiation leads to the appearance of cells with signs of apoptosis: large cells with large vacuoles, a condensed nucleus and bleached chloroplast. The cell sizes did not significantly differ from the control samples and the cell shape became more rounded.

A further area of research is the selection of conditions allowing to increase the yield of carotenoids with minimal loss of microalgae biomass.

\section{References}

[1] O.V. Dymova, T.K. Golovko, Bulletin of the Ufa Scientific Center for Wounds, 3, 4 (2018).

[2] T.J. McQuistan, M.T. Simonich, M.M. Pratt, C.B. Pereira, J.D. Hendricks, R.H. Dashwood, D.E. Williams, G.S. Bailey, Food Chem. Toxicol, 50 (2012).

[3] L. Ambrosino, M. Tangherlini, C. Colantuono, A. Esposito, M. Sangiovanni, M. Miralto, C. Sansone, M.L. Chiusano, Marine Drugs, 17, 10 (2019).

[4] C. Galasso, A. Gentile, I. Orefice, A. Ianora, A. Bruno, D.M. Noonan, C. Sansone, A. Albini, C. Brunet, Nutrients, 11, 6 (2019). 
[5] R. Sathasivam, Ki. Jang-Seu, A Mar Drugs, 16, 1 (2018).

[6] A.M. Lizzul, A. Lekuona-Amundarain, S. Purton, L. Cintra, Biology, 7, 25 (2018).

[7] M. Belkoura, A. Benider, A. Dauta, Annls Limnol, 33, 1 (1997).

[8] L. Gracia, K. Cianca, L. Montero, Sociedad Latinoamericana de biotecnologia ambiental y algal, Florianopolis, Brazil (2015).

[9] N. Politaeva, Y. Smyatskaya, E. Trukhina, F. Ovchinnikov, International Journal of Applied Engineering Research, 12, 21 (2017).

[10] C. Crofcheck, A. Shea, et al., J Biochem Tech, 4, 2 (2012).

[11]Yu.G. Bazarnova, T.A. Kuznetsova, Yu.A. Smyatskaya, Patent for the invention of the Russian Federation, 2695879 (2019).

[12] O.V. Bulda, V.V. Rassadina, G.N. Alekseychuk, N.A. Laman, Plant Physiology, 55, 4 (2018).

[13] S. Nayek, Research Journal of Chemical Sciences, 4, 9 (2014).

[14]A. Tarchevskiy, The main principles of photosynthesis, Edition of the Kazan State University (2017).

[15] G.I. Ali-zade, Modern problems of science and education, 4 (2009).

[16]M.Ya. Shashkina, P.N. Shashkin, A.V. Sergeev, Russian biotherapeutic journal, 4, 8 (2009).

[17]C. Jime'nez, J.M. Capasso, L. Charles, C.L. Edelstein, C.J. Rivard, S. Lucia, S. Breusegem, T. Berl, M. Segovia, Journal of Experimental Botany, 60, 3 (2009).

[18]A. Zuppini, C. Gerotto, B. Baldan, Plant Cell Physiol., 51, 6 (2010). 